PALEO

Revue d'archéologie préhistorique

$15 \mid 2003$

Varia

\title{
L'ours des cavernes de Font-de-Gaume III
}

The cave bear of Font-de-Gaume III

\section{Dominique Armand, Frédéric Plassard et François Prat}

\section{OpenEdition}

Journals

Édition électronique

URL : http://journals.openedition.org/paleo/1303

DOI : $10.4000 /$ paleo.1303

ISSN : 2101-0420

Éditeur

SAMRA

Édition imprimée

Date de publication : 1 décembre 2003

Pagination : 241-244

ISSN : 1145-3370

\section{Référence électronique}

Dominique Armand, Frédéric Plassard et François Prat, «L'ours des cavernes de Font-de-Gaume III », PALEO [En ligne], 15 | 2003, mis en ligne le 03 août 2010, consulté le 07 juillet 2020. URL : http:// journals.openedition.org/paleo/1303 ; DOI : https://doi.org/10.4000/paleo.1303

Ce document a été généré automatiquement le 7 juillet 2020

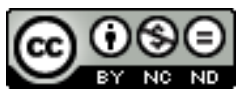

PALEO est mis à disposition selon les termes de la licence Creative Commons Attribution - Pas d'Utilisation Commerciale - Pas de Modification 4.0 International. 


\section{L'ours des cavernes de Font-de- Gaume III}

The cave bear of Font-de-Gaume III

Dominique Armand, Frédéric Plassard et François Prat

1 La grotte de Font-de-Gaume (Les Eyzies, Dordogne, France) est connue depuis sa découverte, en 1901, comme un site d'art pariétal paléolithique de premier ordre. Depuis cette date, plusieurs campagnes de fouille ont été conduites dans cette cavité.

Dans la monographie que publièrent les inventeurs (Capitan, Breuil et Peyrony 1910), un chapitre présente le matériel archéologique recueilli dans les différentes galeries. Quelques lignes sont également consacrées aux ossements d'ours découverts dans le remplissage argileux et qui furent étudiés par F. Harlé.

3 Dans les années soixante, F. Prat entreprend de nouvelles fouilles. Ses travaux ont porté sur deux secteurs principaux :

- Font-de-Gaume I : localisé dans une galerie latérale s'ouvrant à 60 mètres de l'entrée ;

- Font-de-Gaume III : zone d'environ $50 \mathrm{~m}^{2}$, fouillée en vue du réaménagement de la grotte, et située dans la galerie principale entre le «Carrefour » et la « salle des petits bisons ».

Deux niveaux archéologiques principaux y ont été reconnus et ont été décrits par $\mathrm{F}$. Prat et D. de Sonneville-Bordes (1969) :

- la couche 3 est peu riche en faune et le Renne est le taxon le mieux représenté. L'industrie est assez abondante et elle a été attribuée à un Aurignacien typique ;

- en dessous (couches 4-5), l'Ours est l'animal dominant (suivi du Bouquetin, du Cheval, des Bovinés, du Loup et du Cerf) et d'après les dimensions des os longs, il se rapproche d' Ursus spelaeus.

Des restes humains (une dent, à laquelle s'ajoute une phalange qui n'avait pas été décrite précédemment) et quelques vestiges lithiques, parmi lesquels deux pointes de Châtelperron, sont également présents dans ce niveau. 
7 Le matériel des fouilles Prat était conservé, jusqu'à présent, à l'Institut de Préhistoire et de Géologie du Quaternaire de Talence. En prévision de son déménagement au Musée national de Préhistoire des Eyzies, il a semblé opportun de faire un inventaire informatique de cet ensemble. A l'occasion de ce travail, des traces de découpe, qui n'avaient pas été signalées jusqu'alors, ont été observées sur des restes d'Ours des couches $4-5$, nous conduisant à mener une étude plus poussée des restes osseux provenant de ces 2 couches.

8 Les dents d'Ours confirment le diagnostic établi par F. Prat (Prat et Sonneville-Bordes 1969) à partir des éléments post-crâniens à savoir que l'Ours de Font-de-Gaume III s'apparente à Ursus spelaeus.

9 Des stries ont été observées sur 11 côtes (fig. 1 et 2), deux vertèbres thoraciques, un fémur et un humérus. Elles documentent l'exploitation de deux adultes et d'un jeune. Elles sont pour la plupart très nettes et correspondent à des marques de découpe.

Figure 1 - Stries de découpe sur la face externe, près de l'extrémité ventrale, d'une côte d'Ursus spelaeus.

Figure 1 - Cutmarks on the externe side, near the ventral extremity, of an Ursus spelaeus.
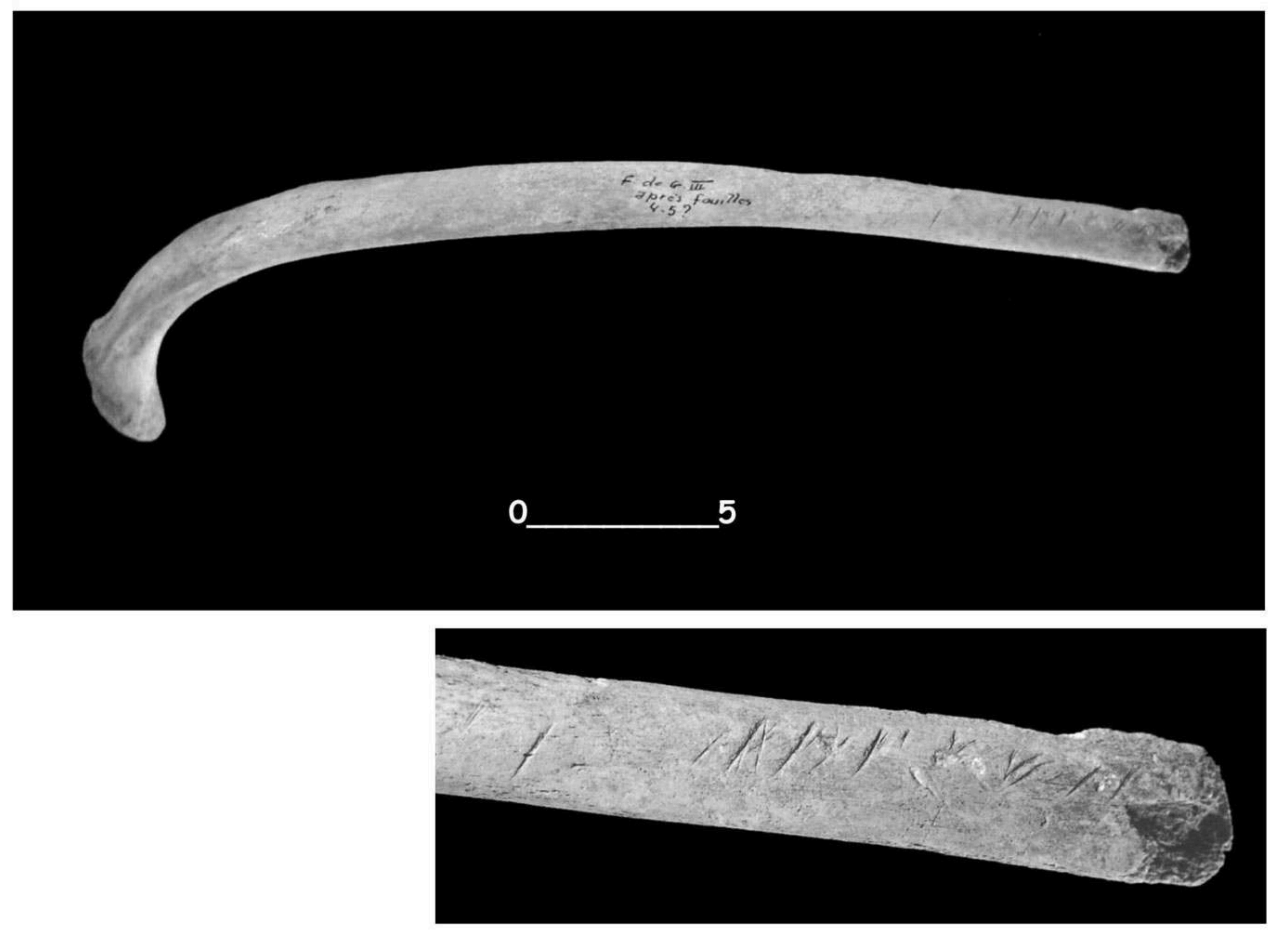
Figure 2 - Stries de découpe sur la face externe dans la portion médiane d'une côte d'Ursus spelaeus. Photo P. Jugie, MNP, Les Eyzies.

Figure 2 - Cutmarks on the externe side, in the middle part, of an Ursus spelaeus.
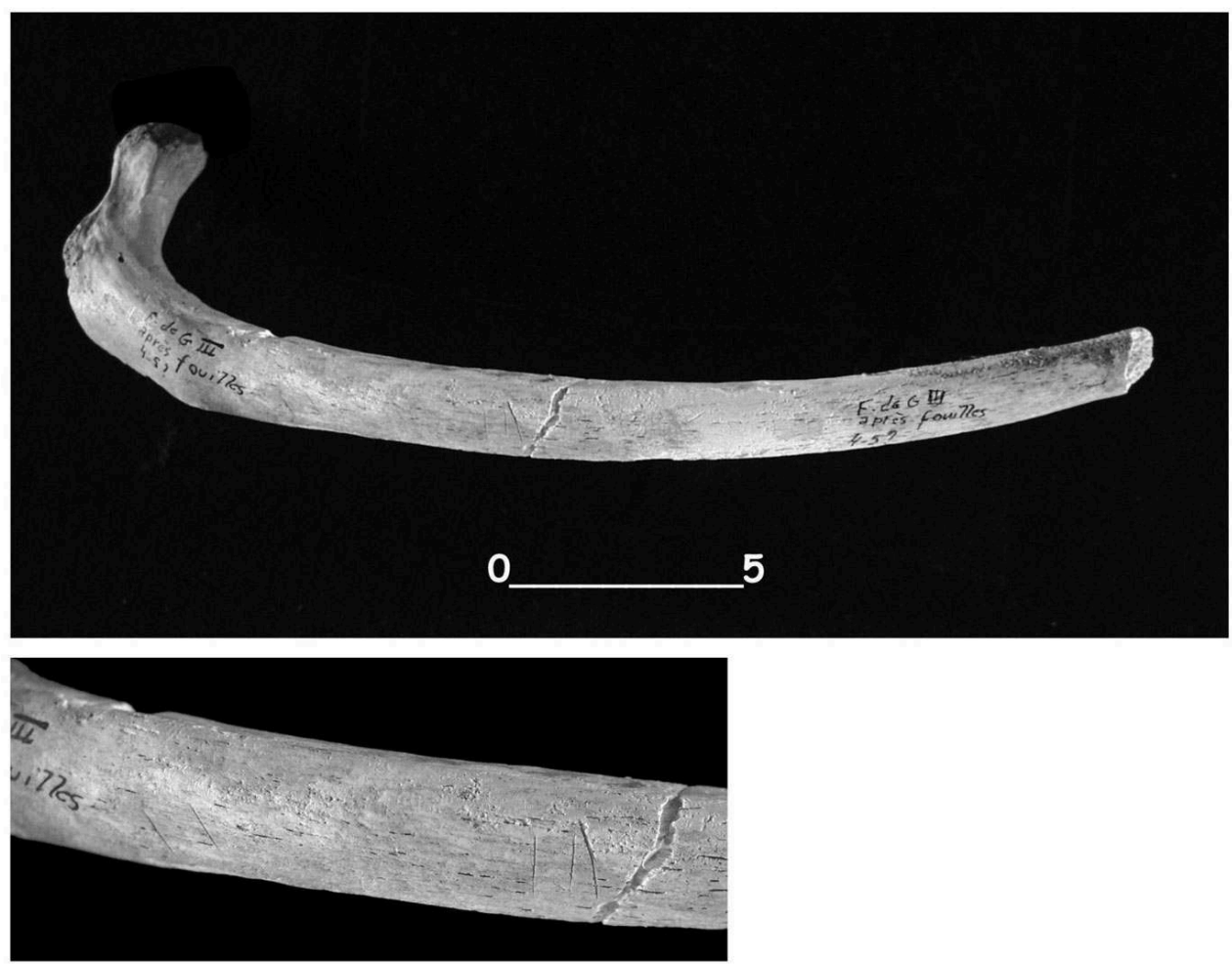

10 Par leur situation presque exclusive sur le squelette axial, ces traces de découpe attestent un comportement d'exploitation peu documenté par ailleurs. En effet, les traces de boucherie, signalées jusqu'alors sur des ossements d'Ours dans d'autres gisements, sont essentiellement situées sur les membres et plus particulièrement dans les portions distales (Auguste 1992 ; David 2002).

11 Pour F. Prat, le fait que les ossements d'Ours de la formation 4-5 soient très souvent entiers ou peu endommagés, et qu'il y ait une forte proportion d'individus jeunes (y compris des nouveaux-nés) permet de penser à une mort naturelle lors de l'hibernation.

Dans ces conditions, les carcasses exploitées n'auraient pas été introduites dans la grotte qui ne semble pas avoir constitué un lieu d'habitat étant donnée la faible quantité de matériel archéologique. Il s'agirait d'une exploitation épisodique dans une grotte à Ours.

La poursuite de l'étude archéozoologique permettra de préciser les modalités exactes de cette exploitation.

14 Soulignons l'intérêt de cette découverte dans un contexte chronologique intéressant, proche de la transition entre le Paléolithique moyen et le Paléolithique supérieur. 


\section{BIBLIOGRAPHIE}

AUGUSTE P. 1992 - Etude archéozoologique des grands mammifères du site pleistocène moyen de Biache-Saint-Vaast (Pas-de-Calais, France) : apports biostratigraphiques et palethnographiques. L'Anthropologie, t. 96, n 1, p. 49-70.

CAPITAN L., BREUIL H. et PEYRONY D. 1910 - La caverne de Font-de-Gaume, aux Eyzies (Dordogne).

Monaco, Imprimerie du Chêne, 271 p.

DAVID F. 2002 - Les ours du Châtelperronien de la grotte du renne à Arcy-sur-Cure, in : TILLET Th. et BINFORD L. R. (sous la dir. de), L'Ours et l'Homme. Actes du Colloque d'Auberives-en-Royans, 1997, ERAUL n 100, Ed. de l'Université de Liège, p. 185-192.

PRAT F. et SONNEVILLE-BORDES D. 1969 - Découvertes récentes de paléolithique supérieur à la grotte de Font-de-Gaume (Dordogne). Quaternaria, XI, Roma, p. 115-132

\section{RÉSUMÉS}

Le niveau 4-5 (fouilles Prat) de la grotte de Font-de-Gaume est riche en ossements d'Ours des cavernes. Quelques artefacts lithiques y ont également été trouvés. Parmi eux, signalons la présence de deux pointes de Châtelperron. La reprise de ce matériel a permis de découvrir des traces de découpe sur des restes d'Ours.

Layer 4-5 (Prat excavation) of Font-de-Gaume cave is reach in cave bear remains. This layer also includes a few lithic artefacts, including two châtelperron points. A recent analysis of this material shows that cutmarks are present on some of the cave bear bones.

\section{INDEX}

Mots-clés : Ursus spelaeus, Châtelperronien, traces de boucherie

Keywords : Ursus spelaeus, Châtelperronien, cutmarks

\section{AUTEURS}

\section{DOMINIQUE ARMAND}

Université Bordeaux I - UMR 5808 - Institut de Préhistoire et de Géologie du Quaternaire. Avenue des Facultés - 33405 Talence cedex.

\section{FRÉDÉRIC PLASSARD}

Université Bordeaux I - UMR 5808 - Institut de Préhistoire et de Géologie du Quaternaire. Avenue des Facultés - 33405 Talence cedex.

\section{FRANÇOIS PRAT}

Université Bordeaux I - UMR 5808 - Institut de Préhistoire et de Géologie du Quaternaire. Avenue des Facultés - 33405 Talence cedex. 